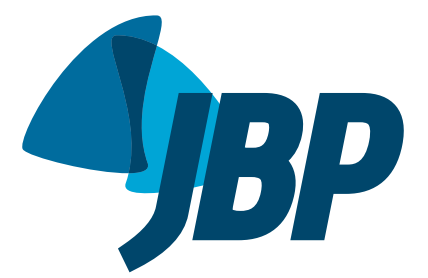

\section{An unusual cause of pleural effusion in a patient with heart failure}

\author{
Daniel Bruno Takizawa',a, Philippe de Figueiredo Braga Colares ${ }^{1, b}$, \\ Olívia Meira Dias,c
}

A 56-year-old female patient with a previous diagnosis of idiopathic dilated cardiomyopathy presented with worsening dyspnea over four days. Her medical history included a diagnosis of xanthogranulomatous pyelonephritis, which had required a left nephrostomy eight months prior to her presentation. On admission, a chest X-ray showed left pleural effusion and cardiomegaly. A sepsis protocol was initiated owing to hypotension and a concern for parapneumonic pleural effusion or empyema. A chest HRCT revealed an atrophic left kidney with multiple hypoattenuating areas with gas bubbles (collections) associated with calculi in the renal pelvis. One of the collections had clear contiguity with the perirenal space next to the diaphragmatic pillar. A diagnostic thoracentesis showed a yellow exudate with low $\mathrm{pH}$ and glucose levels and high lactate dehydrogenase and creatinine levels. The patient was diagnosed with urinothorax and renal abscess, received antibiotic therapy, and underwent total nephrectomy. Urinothorax is a rare cause of pleural effusion, most frequently resulting from obstructive uropathy or iatrogenic/traumatic genitourinary injury..$^{(1-3)}$ Pleural fluid from urinothorax generally reveals a transudate that resolves after removing the urinary tract obstruction. Associated infection leads to pleural fluid with low $\mathrm{pH}$ and glucose levels.

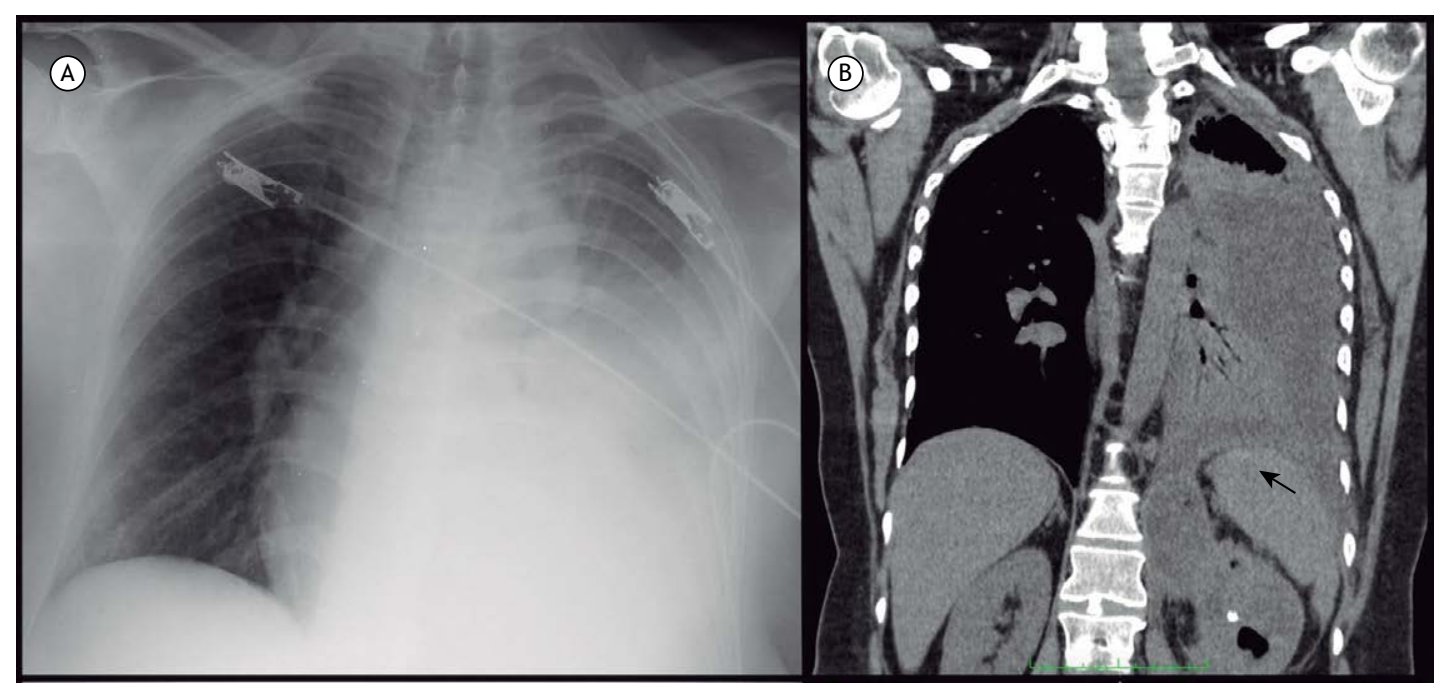

Figure 1. In A, an X-ray of the chest showing extensive left pleural effusion. In $B$, coronal reconstruction from a CT showing the left kidney with a calcified calculus and hypoattenuated areas, one of which draining to the perirenal space and causing left pleural effusion (black arrow).

\title{
REFERENCES
}

1. Toubes ME, Lama A, Ferreiro L, Golpe A, Álvarez-Dobaño JM, GonzálezBarcala FJ, et al. Urinothorax: a systematic review. J Thorac Dis. 2017;9(5):1209-1218. https://doi.org/10.21037/jtd.2017.04.22

2. Garcia-Pachon E, Romero S. Urinothorax: a new approach. Curr Opin Pulm Med 2006;12(4):259-63. https://doi.org/10.1097/01.

\section{mcp.0000230628.65515.86}

3. Ranjan V, Agrawal S, Chipde SS, Dosi R. Urinothorax: A path, less travelled: Case report and review of literature. J Nat Sci Biol Med. 2015;6(1):213-6. https://doi.org/10.4103/0976-9668.149182 